East African Medical Journal Vol. 85 No. 5 May 2008

BURKITT'S LYMPHOMA IN UGANDA: THE ROLE OF IMMUNOHISTOCHEMISTRY IN DIAGNOSIS

R. Lukande, MBChB, MMed, (Path), Lecturer, H.R. Wabinga, MBChB, MMed, MD, Associate Professor and

L.K. Tumwine, MBChB, MMed, Lecturer, Department of Pathology, Faculty of Medicine, Makerere University Medical School,

P.O. Box 7072, Kampala, Uganda

Request for reprints to: Dr. L.K. Tumwine, Department of Pathology, Faculty of Medicine, Makerere University Medical School, P.O. Box 7072, Kampala, Uganda

\title{
BURKITT'S LYMPHOMA IN UGANDA: THE ROLE OF IMMUNOHISTOCHEMISTRY IN DIAGNOSIS
}

\author{
R. LUKANDE, H.R. WABINGA and L.K. TUMWINE
}

\begin{abstract}
Background: Haematoxylin and eosin staining has remained the standard diagnostic method for Burkitt's lymphoma. Ancillary tests including immunohistochemistry, not widely available in developing countries, are important tools in verifying the diagnosis of lymphomas with equivocal morphological findings.

Objective: To evaluate the reliability of haematoxylin and eosin staining in the diagnosis of Burkitt's lymphoma using immunohistochemistry as the gold standard.

Design: Cross sectional study.

Setting: Department of Pathology laboratory, Makerere University Medical School, Uganda. Subjects: One hundred and thirty eight formalin fixed paraffin embedded biopsies of Burkitt's lymphoma diagnosed from January 2001 to December 2005.

Results: Of the 138 tumours, $88.4 \%$ were extra-nodal: jaw $36.2 \%$, ovary $21 \%$, gastrointestinal tract $12.3 \%$ other abdominal $11.6 \%$. Males $(55.8 \%)$ predominated. The sensitivity and specificity of haematoxylin and eosin were $93.2 \%$ and $50 \%$ respectively. The positive and negative predictive values were $91.7 \%$ and $55.6 \%$.

Conclusion: Histology using haematoxylin and eosin staining is sensitive in the diagnosis of Burkitt's lymphoma but not very specific. Immunohistochemical staining with CD20, Ki-67 and bcl-2 is necessary for difficult cases.
\end{abstract}

\section{INTRODUCTION}

Burkitt's lymphoma (BL) is the most common childhood tumour in Uganda (1). The incidence of Burkitt's lymphoma has not changed since the beginning of the HIV / AIDS pandemic in the late eighties (2). In Uganda and many resource poor countries, the diagnosis of Burkitt's lymphoma is still largely based on histological examination using haematoxylin and eosin $(3,4)$. Yet its reliability has not been assessed in Uganda. Notably the diagnostic histological hallmark for Burkitt's lymphoma is the starry-sky pattern formed by the phagocytic histiocytes interspersed among primitive round monomorphic and deeply basophillic/lymphoblasts. This pattern however, is also found in other high grade non-Hodgkin's lymphomas. B-cell Burkitt-like lymphomas appear very similar morphologically and clinically to diffuse large B-cell lymphomas and previous studies using morphology alone have shown that the diagnosis of specific types of non Hodgkin lymphoma could only be accurately made $50-60 \%$ of the time $(5,6)$ using haematoxylin and eosin alone. Ancillary diagnostic tests that include immunohistochemistry, not widely available in 
developing countries, are important tools for verifying the diagnosis of lymphomas presenting with equivocal morphological findings (7). This is further strengthened by using cytogenetic analysis of touch preparations from fresh or frozen tissue for detection of c-myc rearrangements (5).

We have previously reported results of a study in Uganda to assess the reliability of haematoxylin and eosin staining in the diagnosis of Hodgkin's lymphoma and the use of immunohistochemistry in discerning the diagnostic dilemmas of its histopathology (8). We showed that haematoxylin and eosin had a relatively high efficacy and was still recommended for the diagnosis of Hodgkin's lymphoma. However the reliability of haematoxylin and eosin for the diagnosis of Burkitt's lymphoma has not been carried out. We report results of the evaluation of haematoxylin and eosin in the diagnosis of the $\mathrm{BL}$ using iminunohistochemistry as the gold standard.

\section{MATERIALS AND METHODS}

Study design and setting: This laboratory based cross sectional study was carried out in the Department of Pathology, Makerere University to evaluate the reliability of haematoxylin and eosin staining in the diagnosis of Burkitt's lymphoma in Uganda diagnosed during the period 2001 to 2005.

Study population: This consisted of biopsy specimens received and preserved as formalin-fixed, paraffin embedded tissue blocks in the Department of Pathology, Makerere University.

Sampling procedure: One hundred and thirty eight formalin-fixed paraffin embedded biopsies with their corresponding histology reports forms with a diagnosis of Burkitt's lymphoma were retrieved from the archives of the Department of Pathology and consecutively entered into the study. Poorly processed and preserved paraffin blocks and those that were destroyed during storage were excluded.

Morphological diagnosis: Each biopsy was given a unique identification number, sectioned and stained with haematoxylin and eosin and reinterpreted by the investigators (R.L, H.R.W, L.K.T) using criteria of the working formulation for clinical usage (9).

The criteria used for morphological diagnosis of Burkitt's lymphoma were as follows: classical Burkitt's lymphoma was diagnosed when there was cellular cohesiveness, the starry- sky pattern and monomorphic medium sized cells with round nuclei, multiple nucleoli, and a high nucleus to cytoplasmic ratio (Figure 1). Atypical/Burkittlike lymphoma was diagnosed when there was cellular cohesiveness, and/or a starry-sky pattern but composed of medium sized Burkitt's cells with greater pleomorphism in nuclear size and shape. Those that did not have any of the above criteria were classified as other lymphomas.

Figure 1

Photomicrograph of Burkitt's lymphoma showing the starry-sky appearance and strong positivity for CD20 (magnification $x$ 20)

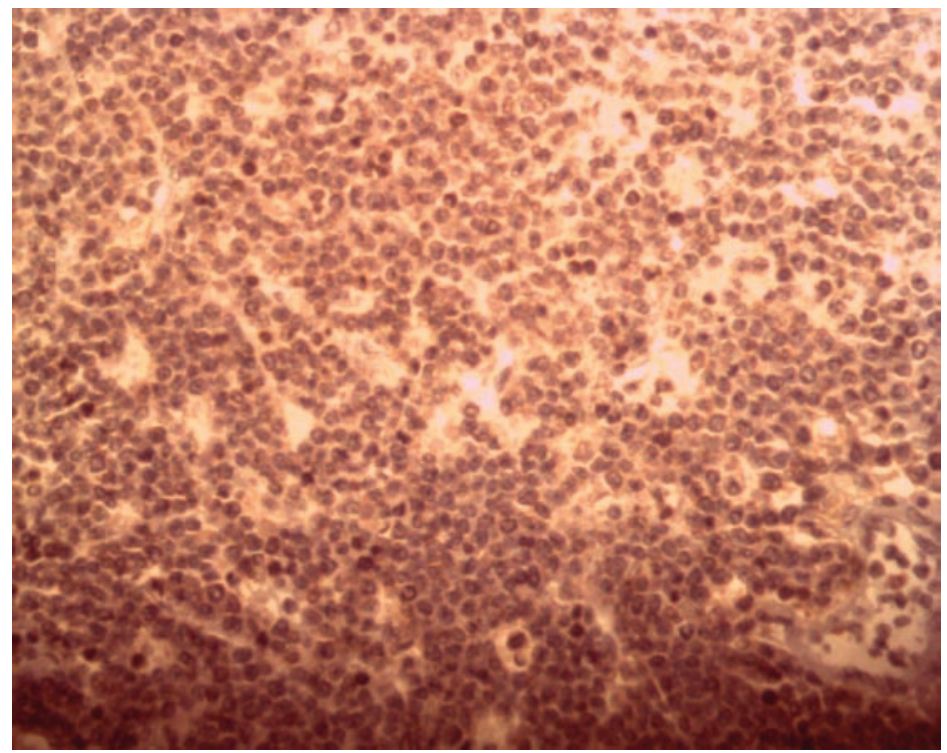


Immunohistochemical analysis: Four- $\mu \mathrm{m}$ thick sections were cut from the blocks and submitted to antigen retrieval using ethylene diamine tetra acetic acid (EDTA) $1 \mathrm{mM}$ ( $\mathrm{pH} 8.0$ ) by micro-waving twice for five minutes at 750 or $900 \mathrm{~W}$. After cooling, the slides were incubated for 30 minutes at room temperature with antibodies against CD20, Bcl-2, and Ki-67 / MIB1. (Details of the antibodies, sources, dilutions and antigen retrieval are in Table 1). The antibodies were detected by using the alkaline phosphatase antialkaline phosphatase immunocomplexes (APAAP) technique (10).

Classical Burkitt's lymphoma and Atypical/ Burkitt-like were diagnosed when they expressed the immunophenotype of CD20 positive, Bcl-2 negative, and a Ki-67 index of $80 \%$ and above (5) (Figure 2).

Study limitation: Cytogenetic analysis using fluorescent in situ hybridisation (FISH) on touch preparations from fresh or frozen tissue to detect presence of c-myc re-arrangements and absence of bcl-2 and bcl-6 re-arrangements that is confirmatory for the diagnosis of Burkitt's lymphoma was not done in this study due to cost and the fact that our department is not yet equipped for cryopreservation.

Data management and analysis: Data were collected and entered into the computer using Epi INFO software (supplied by CDC and WHO) for storage and initial analysis.

Further analysis was done using SPSS software. The data was summarised in frequency tables. For continuous variables such as age, the relevant measures of central tendency were used to explore the data.

The sensitivity, specificity, negative and positive predictive values and the overall Kappa score were calculated using Open Epi software (12).

Table 1

Monoclonal antibodies used in the study

\begin{tabular}{lllll}
\hline Antibody & Clone & Source & Antigen retrieval & Dilution \\
\hline CD20 & L26 & DakoCytomation, CA, USA & EDTA 750 W & $1: 200$ \\
Bcl-2 & 12 & DakoCytomation, CA, USA & EDTA 900 W & $1: 3$ \\
Ki-67 & MIB-1 & DakoCytomation, CA, USA & EDTA 900 W & $1: 20$ \\
\hline
\end{tabular}

Figure 2

Photomicrograph of Burkitt's lymphoma showing Ki-67 positive cells with a proliferation index above $80 \%$ (magnification $\times 20$ )

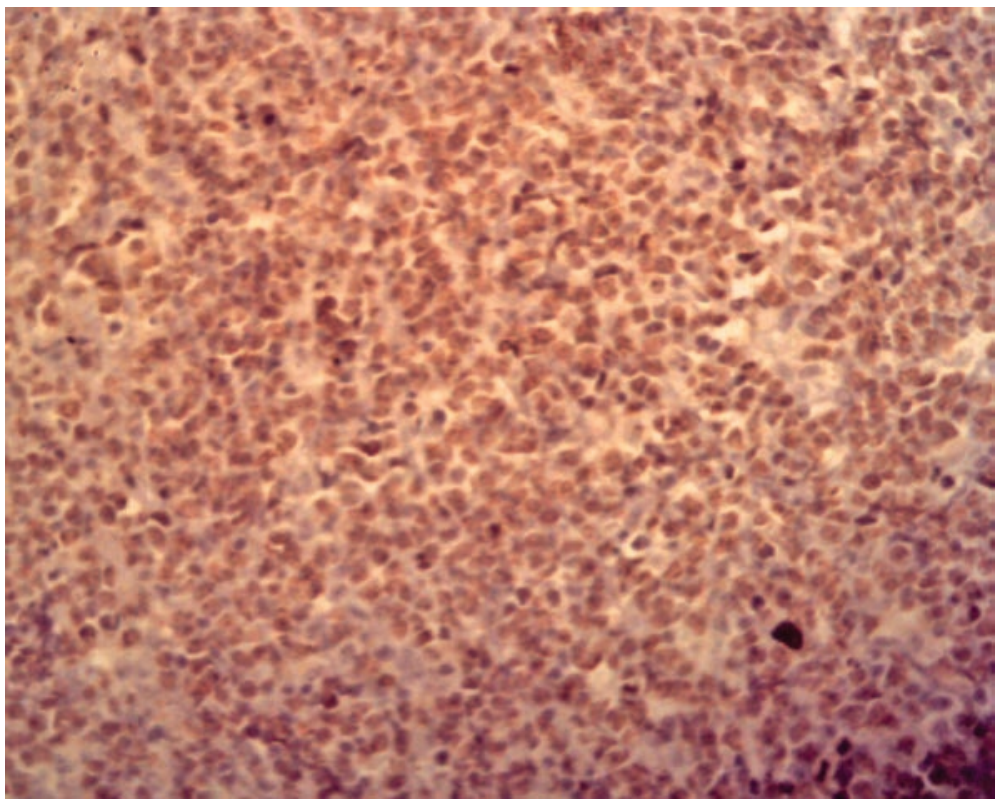




\section{RESULTS}

One hundred and thirty eight cases of Burkitt's lymphoma diagnosed between January 2001 and December 2005 inclusive, in the Department of Pathology, Faculty of Medicine, Makerere University were studied.

The age ranged from 1 to 16 years, with a median of seven years. Seventy seven $(55.8 \%)$ were male, giving a male to female ratio of 1.26:1 (Figure 3).

One hundred and twenty $(87 \%)$ had classical Burkitt's lymphoma; $10(7.2 \%)$ had Atypical / Burkittlike lymphoma and eight (5.8\%) had other diagnoses including two retinoblastomas, two diffuse large B-cell lymphomas and one fibrosarcoma.

The Baganda $36(30 \%)$ were the majority tribe, followed by the Itesot 17 (14.2\%), Basoga $12(10 \%)$, and Lugbara four $(3.3 \%)$. The rest $51(42.5 \%)$ were from 20 other tribes.
One hundred and twenty two (88.4\%) patients had extranodal disease. The jaw accounted for $50(36.2 \%)$, the ovary $29(21 \%)$, gastrointestinal tract $17(12.3 \%)$ and the rest of the abdominal cavity $16(11.6 \%)$.

Immunohistochemical analylss: Of the 138 biopsies, $120(87.0 \%)$ were classical Burkitt's lymphoma, 10 (7.2\%) were Atypical / Burkitt-like lymphoma, and eight $(5.8 \%)$ had other diagnoses (including two retinoblastomas, two diffuse large B-cell lymphomas, one fibrosarcoma, and three others).

Sensitivity, specificity, positive and negative predictive values: The sensitivity, specificity, positive and negative predictive value of haematoxylin and eosin in the diagnosis of BL are shown in Table 2.

Parameters for the reliability of haematoxylin and eosin in the diagnosis of Burkitt's lymphoma are shown in Table 2.

Figure 3

Age distribution of patients with Burkitt's lymphoma

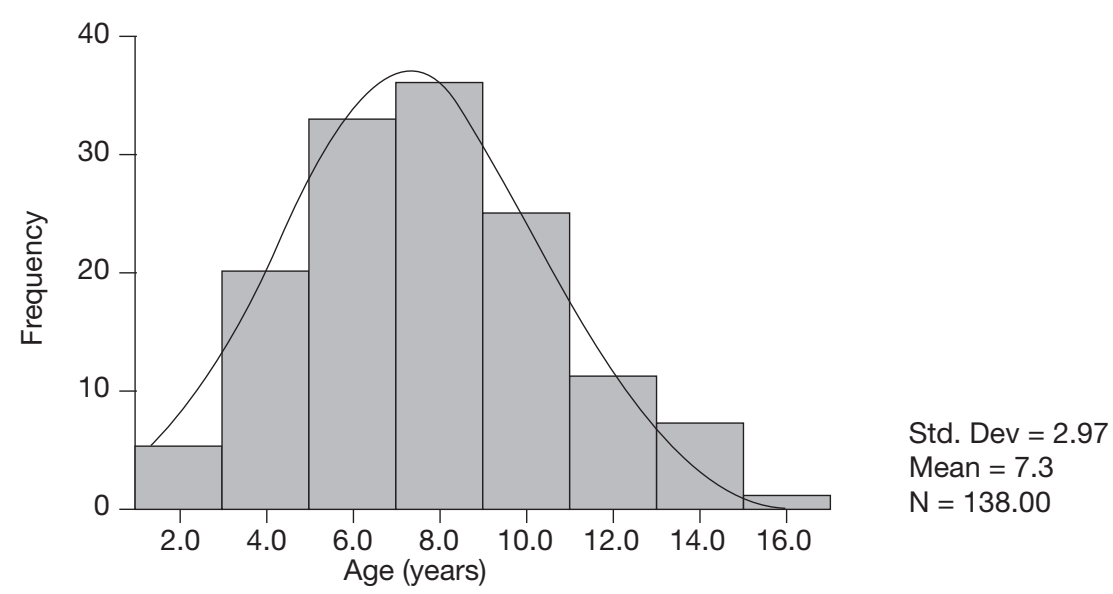

Table 2

Sensitivity, specificity and predictive values of haematoxylin and eosin in the diagnosis of Burkitt's lymphoma, using immunohistochemistry as gold standard

\begin{tabular}{lll}
\hline Parameter & Estimate $(\%)$ & $95 \%$ CIs \\
\hline Sensitivity & 93.2 & $(87.2,96.5)$ \\
Specificity & 50.0 & $(29.9,70.1)$ \\
Positive predictive value & 91.7 & $(85.3,95.4)$ \\
Negative predictive value & 55.6 & $(33.7,75.4)$ \\
Diagnostic accuracy & 87.0 & $(80.3,91.6)$ \\
Likelihood ratio of a positive test & 1.86 & $(1.53-2.27)$ \\
Likelihood ratio of a negative test & 0.14 & $(0.09-0.21)$ \\
Diagnostic odds & 13.8 & $(4.4-42.7)$ \\
Cohen's kappa (un-weighted) & 0.45 & $(0.28-0.62)$
\end{tabular}

CIs $=$ Confidence Intervals 


\section{DISCUSSION}

The aim of the study was to determine the reliability of haematoxylin and eosin in the diagnosis of Burkitt's lymphoma. Specifically, we ascertained the sensitivity, specificity, negative and positive predictive values of haematoxylin and eosin in the diagnosis of Burkitt's lymphoma. The per cent agreement between haematoxylin and eosin staining and immunohistochemistry together with the overall kappa score were also determined.

Distribution of cases by age, sex, tribe and tumour site: Burkitt's lymphoma affected mainly childlren aged 1-16 years and most of them were male. This agrees with similar findings from East Africa $(13,14)$. Most of the patients were Baganda, followed by the Itesot, Basoga, Lugbara and other tribes. The reason for the predominance of the Baganda is due to the fact that Mulago Hospital and Makerere University, where the study was carried out, are located in the central region where the Baganda are the majority tribe. As expected, most of the tumours $(89.2 \%)$ were of extra nodal origin. Most (37.5\%) were from the jaw, the ovary $(24.2 \%)$ and the intestines $(11.7 \%)$ as previously reported by others (14).

Morphological characteristics on haematoxylin and eosin staining: One hundred and twenty $(87 \%)$ had classical Burkitt's lymphoma; 10 (7.2\%) had Atypical /Burkitt-like lymphoma and eight (5.8\%) of the cases previously diagnosed as Burkitt's lymphoma were found to infact have other diseases, two retinoblastomas, two diffuse large B-cell lymphomas and one fibrosarcoma.

Immunohistochemical characteristics: In this study, Burkitt's lymphoma had a Ki-67 proliferation index that ranged from $80 \%$ to $100 \%$ in $112(93.3 \%$ ) cases. This agrees with what Haralambieva et al (15) and Frost et al (16) found: a mean of Ki-67 of 99\% in Burkitt's lymphoma. Hutchison and others found Ki-67 to be diffusely reactive in all the 41 paediatric B-cell lymphomas studied that included 13 Burkitt's lymphomas (17). The immunohistochemical staining characteristics of Burkitt's lymphoma in this study showed a CD20 positive rate in $119(99.2 \%)$. These results are similar to those by Carbone et al (18) who also found that 40 out of $41(97.6 \%)$ of Burkitt's lymphoma were CD20+.
Bcl-2 is rarely expressed in Burkitt's lymphoma (18). The bcl-2 antibody staining results in this study was positive in one $(0.8 \%)$ of Burkitt's lymphoma compared to $50 \%$ in other lymphomas. The study findings conform to what Frost et al (16) found where bcl-2 was positive in $4 \%$ of Burkitt's lymphoma versus 37\% in diffuse large B-cell lymphoma in a study of 33 Burkitt's lymphomas and 20 diffuse large B-cell lymphomas.

Sensitivity and specificity: The sensitivity of haematoxylin and eosin in this study was $93.2 \%$, which is high, and therefore makes it a good screening test for Burkitt's lymphoma. In a study by the International Lymphoma Study Group (19) the sensitivity was found to be $85 \%$ for most of the major lymphoma types. Haralambieva et al (15) found an accuracy of $100 \%$ in the analysis of ten reference paediatric Burkitt's lymphomas. The findings in the current study are similar to those of the above studies. Furthermore a previous study in Uganda (8) showed sensitivity of haematoxylin and eosin staining of $76 \%$ in Hodgkin's lymphoma whereas the International Lymphoma Study Group (19) in a study on a number of lymphomas found a slightly higher sensitivity of $85 \%$. This was lower than what was found in the current study. The reason for these differences may be because Hodgkin and other nonHodgkin's lymphomas are more heterogeneous in both morphology and immunophenotype than Burkitt's lymphoma (20).

The specificity in this study was low at $50 \%$. This means that haematoxylin and eosin is not very good at picking out those that do not have Burkitt's lymphoma. In fact eight $(5.8 \%)$ of the tumours in this study that had previously been diagnosed as Burkitt's lymphoma were found to have other diagnoses: two retinoblastomas, two diffuse large cell lymphomas and one fibro sarcoma. The study findings are similar to what the International Lymphoma Study Group had found in previous studies (19) with specificity levels of 50\% to $60 \%$.

Positive and negative predictive values: The positive predictive value of haematoxylin and eosin in the diagnosis of Burkitt's lymphoma was $91.7 \%$ is the probability of a pathologist correctly diagnosing Burkitt's lymphoma while the negative predictive value was $55.6 \%$. This means that the probability of a pathologist excluding Burkitt's lymphoma is 
only $55.6 \%$. These results are comparable to those of our previous study on Hodgkin's lymphoma in Uganda (8).

Level of agreement between the two tests: The per cent agreement between haematoxylin and eosin and immunohistochemistry as a gold standard was $87 \%$. Lones et al (21) in a study of 200 B-cell lymphomas that included Burkitt's lymphoma and high grade Burkitt-like lymphoma found a per cent agreement of $88 \%$ for Burkitt's lymphoma. The overall Cohen's Kappa score of 0.45 was fairly good since it lies between 0.4 and 0.75 .

In conclusion, haematoxylin and eosin staining had a sensitivity of $93.2 \%$, a specificity of $50 \%$, and overall Cohen's Kappa statistic of 0.45 . It has therefore got a fairly high efficacy in the diagnosis of Burkitt's lymphoma and is still recommended. However immunohistochemistry is necessary for cases with equivocal morphological findings.

\section{REFERENCES}

1. Burkitt, D. The discovery of Burkitt's lymphoma. Cancer. 1983; 51: 1777-1786.

2. Parkin, M., Garcia-Giannoli, H., Raphael, M., et al. Non Hodgkin lymphoma in Uganda: a case-control study. AIDS. 2000; 14: 2929-2936.

3. Cool, C. and Bitter, M. The malignant lymphomas of Kenya; Morphology, immunophenotype, and frequency of EBV in 73 cases. Hum. Pathol. 1997; 28: 1026-1033.

4. Lazzi, S., Ferrari, F., Nyongo, A., et al. HIV-associated malignant lymphomas in Kenya (Equatorial Africa). Human Pathol. 1998; 29: 1285-1289.

5. Ferry, J. Burkitt's lymphoma: Clinicopathologic features and differential diagnosis. Oncologist. 2006; 11: 375-383.

6. Whitcomb, C., Crissman, J. and Flint, A. Reproducibility of morphologic classification of non-Hodgkin's lymphomas using the Lukes-Collins system. The Southeastern Cancer Study Group experience. Amer. J. Clin. Pathol. 1983; 82: 383-389.

7. Pileri S. and Sabattini, E. A rational approach to imumunohistochemical analysis of malignant lymphomas on paraffin wax sections. J. Clin. Pathol. 1997; 50: 2-4.

8. Tumwine, L., Wabinga, H. and Odida, M. Haematoxylin and eosin staining in the diagnosis of Hodgkin's disease in Uganda. East Afr. Med. J. 2003; 80: 119-123.
9. Armitage, J.O. Staging non-Hodgkin lymphoma. CA Cancer J. Clin. 2005; 55: 368-376.

10. Sabattini, E., Bisgaard, K. and Ascani, S. Envision Plus: A new immunohistochemical method of choice for diagnostics and research. Critical comparison with the APAAP, ChemMate, CSA, LABC and SABC techniques. J. Clin. Pathol. 1998; 51: 506-511.

11. SPSS [computer program]. Version. Chicago, Illinois 60606: SPSS Inc. Headquarters, 233 S.

12. Anon. Open Source Statistics fom Public Health. http:// www.openepi.com/Menu/OpenEpi; 2007.

13. Burkitt, D. A sarcoma involving the jaws in African children. Brit. J. Surg. 1958; 46: 218-223.

14. Mwanda, O., Rochford, R., Moormann, A., et al. Burkitt's lymphoma in Kenya: Geographical, age, gender and ethnic distribution. East Afr. Med. J. 2004; 8: 568-577.

15. Haralambieva, E., Boerma, E. and van Imhoff, G. Clinical, immunophenotypic, and genetic analysis of adult lymphomas within morphologic features of Burkitt's lymphoma. Amer. J. Surg. Pathol. 2005; 8: 1086-1094.

16. Frost, M., Newell, J. and Lones, M. Comparative immunohistochemical analysis of pediatric Burkitt's lymphoma and diffuse large B-cell lymphoma. Amer. J. Clin. Pathol. 2004; 121: 384-392.

17. Hutchison, R., Finch, C. and Kepner, J. Burkitt's lymphoma is immunophenotypically different from Burkitt-like lymphoma in young persons. Ann. Oncol. 2000; 11: 35-38.

18. Carbone, A., Gloghini, A. and Gaidano, G. AIDS related Burkitt's lymphoma. Amer. J. Clin. Pathol. 1995; 103: 561-567.

19. Anon. The non-Hodgkin's lymphoma classification project (1970). A clinical evaluation of the International Lymphoma Study Group Classification of nonHodgkin's Lymphoma. Blood. 1970; 89: 3909-3918.

20. Chan, J., Banks, P. and Clearly, M. A revised EuropeanAmerican classification of lymphoid neoplasms proposed by the International Lymphoma Study Group. A summary version. Amer. J. Clin. Pathol. 1995; 103: 543-560.

21. Lones, M., Aspirin, A. and Raphael, M. Mature B-cell lymphoma/leukemia in children and adolescents: Intergroup pathologist consensus with the Revised European-American Lymphoma Classification. Ann. Oncol. 2000; 11: 47-51. 